\title{
Isolierung des „Dactylins“ von indischen Matthiola Incana, R. Br.
}

\author{
Von Aziz Ur Rahman und M. Sami Khan \\ Departamento de Química e Ingeniería Química, Universidad Nacional del Sur, \\ Bahía Blanca (Argentinien) \\ (Z. Naturforschg. 17 b, 9-11 [1962] ; eingegangen am 31. Juli 1961)
}

\begin{abstract}
Die Isolierung des Dactylins in einer Ausbeute von 0,4\% als ein Aggregat gelblicher Nadeln, Schmp. $187-189^{\circ}$, von indischen Mathiola Incana, R. Br., wird beschrieben. Die Identität des Glykosids mit Isoramnetin-3.4-di-glucosid wird durch Totale Hydrolyse des Glykosids zu Isoramnetin, Schmp. $303-305^{\circ}$, und Glucose einerseits und die vollständige Methylierung des Glykosids und die darauffolgende Hydrolyse zu 3.5.7-Trimethoxy-3.4-di-hydroxy-flavon, Schmp. 206-207 ${ }^{\circ}$, andererseits, bestätigt.
\end{abstract}

Todri ${ }^{1-3}$, eine indische Pflanze von medizinischer Bedeutung, obwohl nicht in Indien beheimatet, wird in den Gärten von Nord-Indien angebaut. Man unterscheidet gewöhnlich in Indien dreierlei Todri-Arten, nämlich die „rote“, Cheiranthus Cheiri, Linn, die „weiße“, Matthiola Incana, R. Br. und die gelbe Varietät für welche noch kein botanischer Name bekannt gemacht wurde; alle drei gehören zur Familie der Cruciferen.

Die Samen, Blüten und Blütenblätter aller in Indien vorkommenden Todri-Arten werden als Stimulantia und Aphrodisiaca angewendet ${ }^{4}$.

Die einzigen Angaben über flavonoide Substanzen der genannten Pflanze findet man in der Arbeit von Perkin und Hummel ${ }^{5}$ über die englische „Yellow wall Flower", Cheiranthus Cheiri, Linn. Diese Autoren berichteten über die Isolierung des Quercetins sowie des Iso-Ramnetins (I) durch direkte hydrolytische Spaltung des rohen Produktes aus dem alkoholischen Pflanzenextrakt. Die vorliegende Arbeit berichtet über die Isolierung des Dactylins (II), d. h. des Isoramnetin-3.4-di-glucosids aus dem Samen des sogenannten weißen Todris, Matthiola Incana, R. Br. aus Indien. Die Isolierung des (II) aus den Blütenstäuben des Obstgarten-Grases (Phleum Pratense) wurde bereits früher von INGLetT ${ }^{6}$ beschrieben.

(II) wurde nunmehr in einer Ausbeute von $0,4 \%$ als ein Aggregat gelblicher Nadeln, Schmp. 187 bis $189^{\circ}$ isoliert. Das Glykosid wurde durch Fraktionierung des alkoholischen Extraktes mittels neutralem

1 A. K. Nadkarni, Indian Materia Medica, 1954, S. 304.

2 Wealth of India, C.S.I.R., India, Band II, 1950, S. 127.

3 R. N. Chopra u. I. C. Chopra, Glossary of Indian Medicinal Plants, 1956, S. 60.

4 Kirtikar u. Basu, Indian med. Plants, 1918, S. 87. sowie basischem Bleiacetat isoliert. Auf diese Art gewinnt man das Glykosid als ein reines, gelbes Produkt.

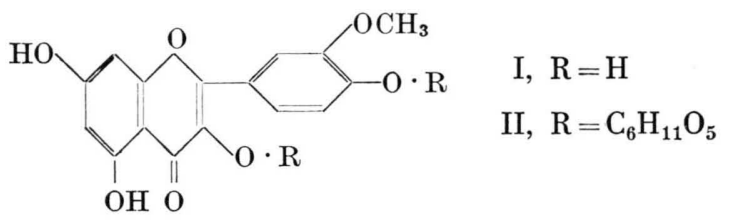

Die Totalhydrolyse des Dactylins mit 7-proz. Schwefelsäure gab (I) ${ }^{7,8}$ als gelbe Nadeln, Schmp. $303-305^{\circ}$; unter Zersetzung. Das Acetylderivat von (I) schmolz bei $203-205^{\circ}$ und durch Behandlung mit Di-methyl-sulfat bekam man ein Methylderivat, mit dem Schmp. 146-147 .

Die vollständige Methylierung von (II) und die darauffolgende Hydrolyse mit 7-proz. Schwefelsäure lieferte eine Substanz vom Schmp. 206-207 ${ }^{\circ}$. Dieser Schmelzpunkt wurde von INGLETT ${ }^{6}$ als der des 3.5.7-Tri-methoxy-3.4-di-hydroxy-flavons beschrieben.

Der Zuckerbestandteil wurde als Glucose identifiziert (Osazon) und $R_{f} 0,17$ in einem aufsteigenden Chromatogramm in $\mathrm{BuOH}: \mathrm{AcOH}: \mathrm{H}_{2} \mathrm{O}(4: 1: 5)$.

Die UV-Absorptionsspektren von (I) und (II) in Äthanol zeigten Maxima bei $255 \mathrm{~m} \mu(\log \varepsilon 4,15)$ und $375 \mathrm{~m} \mu(\log \varepsilon 4,19)$, bzw. bei $253 \mathrm{~m} \mu(\log \varepsilon$ 4,25), $267 \mathrm{~m} \mu(\log \varepsilon 4,25)$ ab $347 \mathrm{~m} \mu(\log \varepsilon 4,19)$, und zeigten dabei eine große Ähnlichkeit zu den Absorptionsmaxima die schon früher für $(\mathrm{I})^{6}$ beschrieben wurden. $(256,375 \mathrm{~m} \mu: \log \varepsilon 4,01$ und

5 A. G. Perkin u. J. J. Hummel, J. chem. Soc. [London] 69, 566 [1896].

${ }^{6}$ G. E. IngLett, Nature [London] 178, 1346 [1956] ; J. org. Chemistry 23, 93 [1958].

7 T. Heap u. R. Robinson, J. chem. Soc. [London] 1926, 2336.

8 KuHN u. Löw, Chem. Ber. 77, 196 [1944]. 
$4,32)$ für (II) ${ }^{6}(252,262,340 \mathrm{~m} \mu: \log \varepsilon 4,29$, $4,31,4,15)$. Die hydroxyl- und carbonyl-infraroten Frequenzen von (I) und (II) in festem Zustande (KBr-Tabletten) fand man bei $2,97 \mu, 3,01 \mu(\mathrm{OH})$ und $6,04 \mu, 6,04 \mu$ (CO.)

\section{Beschreibung der Versuche}

\section{Extrahierung des Dactylins}

An der Luft getrocknete, fein gemahlene, weiße Todri-Samen $(500 \mathrm{~g})$ wurden durch sorgfältige erschöpfende Extrahierung mit Petroläther (Sdp. $40-60^{\circ}$ ) entfettet. Das entfettete Mehl wurde dreimal mit siedendem Äthylalkohol extrahiert und dabei wurde jedesmal mit 2,5 $l$ Äthanol $8 \mathrm{Stdn}$. lang mit Rückfluß gekocht. Der gesamte Extrakt, im Vakuum konzentriert, lieferte eine dicke braune Masse von pastenartiger Konsistenz. Diese wurde hintereinander mit Tetrachlorkohlenstoff und Äthyläther behandelt, um jegliche hinterbleibende fettartige Substanz sowie jegliche anwesende freie Flavone zu entfernen. Der ätherische Extrakt zeigte nach seiner Untersuchung keine freien Flavone. Der ätherunlösliche Rest war eine braune, zähe, pastenartige Masse; Ausbeute 25 Gramm (5 Prozent).

$$
\begin{gathered}
\text { Fraktionierung } \\
\text { des Roh-Glykosidgemischs }
\end{gathered}
$$

Das rohe Glykosid $(25 \mathrm{~g})$ wurde in etwa einem $l$ Äthylalkohol gelöst und einige wenige $\mathrm{cm}^{3}$ heiße, neutrale, alkoholische Bleiacetatlösung hinzugegeben. Die gelbliche, fettig aussehende, präzipitierte Masse, die sich dabei abschied, wurde abfiltriert. $\mathrm{Zu}$ dem heißen Filtrat wurde tropfenweise soviel neutrale, essigsaure Bleilösung zugegeben, bis sich kein Präzipitat mehr abschied. Der orangefarbige Bleikomplex (A) wurde abfiltriert und mit heißem Äthanol gewaschen um es von jedem anderen glykosidischen Stoff zu befreien. Das Filtrat wurde gekocht. $\mathrm{Zu}$ der siedenden Lösung gab man solange eine alkoholische Lösung basischen Bleiacetats hinzu, bis sich kein Präzipitat mehr abschied. Der gelb gefärbte Bleikomplex (B) wurde filtriert und gewaschen. Der Überschuß an Blei, der sich in dem Filtrat befand, wurde mit Schwefelwasserstoff gänzlich gefällt. Das Präzipitat wurde filtriert und gründlich mit reinem, heißem Äthanol ausgewaschen. Das Filtrat wurde bis auf $15 \mathrm{~cm}^{3}$ unter geringem Druck konzentriert. Dies gab eine negative Reaktion auf etwaige flavonoide Substanzen.

$$
\text { Zersetzung des Bleikomplexes }
$$

Der orangefarbige Bleikomplex (A) wurde zersetzt. Das alkoholische Filtrat wurde gekocht, um jeden Überschuß an Schwefelwasserstoff zu beseitigen und schließlich bis auf etwa $60 \mathrm{~cm}^{3}$ im Vakuum konzentriert. Das Konzentrat wurde bis zu etwaiger Kristallisation stehengelassen, doch selbst nach mehreren Wochen schied sich keine kristalline Substanz ab.
Zersetzung des Bleikomplexes

Der gelb gefärbte Bleikomplex (B) wurde noch in feuchtem Zustande in heißem Äthylalkohol aufgeschlämmt und wie oben zersetzt; das Filtrat wurde im Vakuum konzentriert. Das äthanolische Konzentrat $\left(50 \mathrm{~cm}^{3}\right)$ schied, nachdem es mehrere Tage stand, ein gelbes, kristallines Produkt ab, Schmp. $187-189^{\circ}$.

Nach Reduktion mit Magnesium und Salzsäure zeigte es einen rosafarbigen Ton. Es löste sich in heißem Äthylalkohol ganz auf mit gelber Farbe und gab ein gelbes Salz mit basischem Bleiacetat. Es gab eine gelbe Lösung mit einer 5-proz. Natriumcarbonat-Lösung sowie mit einer 1-proz. wässerigen Natronlauge. Der $R_{f}$-Wert 0,48 bei $20{ }^{\circ} \mathrm{C}$ wurde in einem aufsteigenden Chromatogramm auf Whatman Papier Nr. 1 bestimmt. $\mathrm{Als}$ Irrigations-Lösungsmittel wurde das Gemisch $\mathrm{BuOH}: \mathrm{AcOH}: \mathrm{H}_{2} \mathrm{O}(4: 1: 5)$ verwandt.

$$
\begin{array}{lllll}
\mathrm{C}_{27} \mathrm{H}_{30} \mathrm{O}_{17} \cdot \mathrm{H}_{2} \mathrm{O} & \text { Ber. } & \text { C } 49,66 & \text { H } 5,09 . \\
& \text { Gef. } & \text { C } 49,08 & \text { H } 5,67 .
\end{array}
$$

Totale Hydrolyse des Glykosids

$$
\text { (D a ctylin) }{ }^{1}
$$

Das Glykosid (1,5 g) wurde 2 Stdn. unter Rückfluß mit $225 \mathrm{~cm}^{3}$ 7-proz. wässeriger Schwefelsäure behandelt. Das gelbe, sich hierbei abscheidende Aglykon wurde filtriert, gut mit Wasser gewaschen und getrocknet; Ausbeute 1,2 Gramm. Es kristallisierte aus verdünntem Äthanol in Form von gelben Nadeln (Schmp. mit eintretender Zersetzung $303-305{ }^{\circ} \mathrm{C}$. Es zeigte nach Reduktion mit Magnesium und Salzsäure eine rosa Farbe. Der $R_{f}$-Wert, bei $20^{\circ} \mathrm{C}$ in einem aufsteigenden Chromatogramm auf Whatman-Papier Nr. 1 bis auf $29 \mathrm{~cm}$ mit der Mischung $\mathrm{BuOH}: \mathrm{AcOH}: \mathrm{H}_{2} \mathrm{O}$ (4: $\left.: 1: 5\right)$, wurde zu 0,92 gefunden.

$$
\begin{aligned}
& \mathrm{C}_{16} \mathrm{H}_{12} \mathrm{O}_{7} \text { (Isorhamnetin) } \\
& \begin{array}{llllll}
\text { Ber. } & \text { C } 60,81 & \text { H } 3,83 & \mathrm{OCH}_{3} & 9,8 \text {. } \\
\text { Gef. } & \text { C } 60,50 & \text { H } & 3,62 & \text { OCH, }_{3} & 9,4 \text {. }
\end{array}
\end{aligned}
$$

\section{A c e t a t}

Das Aglykon (0,3 g) wurde unter Rückfluß mit Essigsäureanhydrid $\left(18 \mathrm{~cm}^{3}\right)$ und Pyridin $\left(4 \mathrm{~cm}^{3}\right)$ 4 Stdn. gekocht. Die bei der Reaktion entstandene Mischung wurde nach ihrer Abkühlung auf zerkleinertes Eis gegossen. Der feste Stoff, der sich hierbei abschied, wurde filtriert, aus verdünntem Äthanol umkristallisiert (Behandlung mit Tierkohle). Es ergaben sich farblose Nadeln (Schmp. $203-205^{\circ} \mathrm{C}$ ).

$$
\begin{aligned}
& \mathrm{C}_{24} \mathrm{H}_{20} \mathrm{O}_{11} \text { (Isorhamnetintetraacetat) } \\
& \text { Ber. C } 59,57 \text { H 4,16. } \\
& \text { Gef. C 59,14 H 4,00. }
\end{aligned}
$$

Das Tetraacetyl-Derivat wurde durch 1-stdg. Erhitzen unter Rückfluß mit Äthanol und Salzsäure $(1: 1)$ seiner Deacetylierung unterworfen.

Das so wiedergewonnene Aglykon wurde aus verdünntem Äthanol umkristallisiert (gelbe Nadeln, alleine sowie im Gemisch mit dem originalen Aglykon schmelzend bei $\left.303-305{ }^{\circ} \mathrm{C}\right)$. 
Methyläther

(3.4.7-Tri-methoxy-isorhamnetin)

Aglykon $(0,4 \mathrm{~g})$ in trockenem Aceton $\left(80 \mathrm{~cm}^{3}\right)$ wurde mit Dimethylsulfat $\left(2 \mathrm{~cm}^{3}\right)$ und wasserfreiem Kaliumcarbonat $(6,4 \mathrm{~g}) 30$ Stdn. unter Rückfluß erhitzt. Die Lösung wurde nach dem Erkalten filtriert und der Rückstand, erhalten durch Entfernung des Lösungsmittels von dem Filtrat, ergab, aus Äthanol kristallisiert (Tierkohle), farblose Nadeln, Schmp. $146-147^{\circ} \mathrm{C}$.
$\mathrm{C}_{19} \mathrm{H}_{18} \mathrm{O}_{7}$
Ber.
C 63,74
H 5,07 .
Gef. C 63,5
$\mathrm{H} 5,00$.

Vollständige Methylierung und darauffolgende Hydrolyse des Glykosids

Ein Gemisch aus 0,4 $\mathrm{g}$ des Glykosids, $80 \mathrm{~cm}^{3}$ Aceton, $2 \mathrm{~cm}^{3}$ Dimethylsulfat und 4,8 $\mathrm{g}$ frisch gebranntes reines Kaliumcarbonat wurde $50 \mathrm{Stdn}$. unter Rück- fluß und etwas Schütteln erhitzt. Die Mischung wurde abgekühlt, filtriert und der Rückstand mit heißem Aceton gewaschen. Der nach dem Verdampfen des Filtrats überbleibende Rest wurde mehrere Male mit Petroläther gewaschen, um etwaigen Überschuß an Dimethylsulfat zu beseitigen und schließlich mit 7-proz. Schwefelsäure $2 \mathrm{Stdn}$. unter Rückfluß hydrolytisch gespalten. Das in dieser Reaktion entstandene Produkt wurde in einem Eisbad gekühlt und der abgeschiedene feste Stoff filtriert, gewaschen und getrocknet; die Ausbeute betrug $0,2 \mathrm{Gramm}$. Er kristallisierte aus Äthanol (Tierkohle) in gelben Nadeln (Schmp. $206-207^{\circ} \mathrm{C}$ )
$\mathrm{C}_{18} \mathrm{H}_{16} \mathrm{O}_{7}$
Ber. C 62,84
Gef $C 62,60-\mathrm{H} 4,50$.

Herrn Prof. Dr. H. Dannenberg, München, danken wir herzlich für die UV- und IR-Spektren, dem "C o n sejo Nacional de Investigaciones Científicas y Técnicas, Buenos Aires" für die wirtschaftliche Förderung der Arbeit.

\title{
Innerkristalline Quellung und Schichteinschlußverbindungen von Calcium-mono-methyl- und Calcium-mono-äthylphosphorsäureester ${ }^{1}$
}

\author{
Von Kurt Hartl und Armin Weiss
}

Eduard-Zintl-Institut der Technischen Hochschule Darmstadt

(Z. Naturforschg. 17 b, 11-14 [1962]; eingegangen am 20. September 1961)

Calcium-mono-methyl- und Calcium-mono-äthylphosphorsäureester

$$
\left\{\begin{array}{l}
\left.\mathrm{Ca} \mathrm{PO}_{4} \mathrm{R} \cdot \mathrm{H}_{2} \mathrm{O}\right\} \\
\text { Calciumphosphatschicht }
\end{array} \underset{\text { Quellungsflüssigkeit }}{\mathrm{X}}\right.
$$

bilden orthorhombische Schichtengitter und sind zu einer begrenzten eindimensionalen innerkristallinen Quellung mit Wasser, Ammoniak, Blausäure, Alkoholen, Phenolen, Äthern, Ketonen, Aminen und Carbonsäuren befähigt. Beim Abbau der unter Alkohol gequollenen Proben lassen sich definierte Zwischenstufen der Zusammensetzung

$\left\{\mathrm{Ca} \mathrm{PO}_{4} \mathrm{R} \cdot \mathrm{H}_{2} \mathrm{O}\right\} \cdot 0,5 \mathrm{ROH}$

gewinnen, die als Schichteinschlußverbindungen aufzufassen sind.

Aus den sekundären Calciumsalzen der Monomethyl- und Mono-äthylphosphorsäureester ${ }^{2,3}$ mit der Bruttoformel $\mathrm{CaPO}_{4} \mathrm{C}_{\mathrm{n}} \mathrm{H}_{2 \mathrm{n}+1} \cdot 2 \mathrm{H}_{2} \mathrm{O}$ kann eine der beiden Wassermolekeln z. B. durch längeres Erwärmen auf $50{ }^{\circ} \mathrm{C}$ entfernt werden, ohne daß hierbei die Kristalle makroskopisch zerstört werden oder die Schärfe der Röntgeninterferenzen nennenswert beeinträchtigt wird. Bei Raumtemperatur wird das abgegebene Wasser wieder aufgenommen. Die zweite Wassermolekel ist wesentlich fester gebunden. Bei ihrer Entfernung bleiben opake Pseudomorphosen zurück, die nur mehr eine einzige scharfe Röntgeninterferenz liefern.

1 Vgl. hierzu K. Hartu u. A. Weiss, Angew. Chem. 71, 379 [1959].
Die röntgenographische Untersuchung von Pulverpräparaten und Einkristallen zeigte, daß beide Estersalze orthorhombisch kristallisieren. Zwei Gitterdimensionen sind bei beiden Salzen gleich und unabhängig davon, ob die Formeleinheit zwei Wassermolekeln oder nur eine enthält. Die dritte Gitterdimension ändert sich mit der Größe des Alkylrestes und mit dem Wassergehalt.

Daraus wird wahrscheinlich, daß in diesen Verbindungen Schichtengitter vorliegen, worauf bereits der blättrige Habitus der Kristalle einen Hinweis gibt. Die Schichtabstands-Änderung mit der Entfernung von einem Mol Wasser pro Formeleinheit

\footnotetext{
2 J. Cavalier, Bull. Soc. chim. France 19, 883 [1898]; Ann. chim. phys. 18, 453 [1899].

3 K. Langheld, Ber. dtsch. chem. Ges. 44, 2076 [1911].
} 